\title{
AN AFFORDABLE INSULIN PUMP FOR TYPE-1 DIABETIC PATIENTS: A CASE STUDY OF USER-IN-THE-LOOP APPROACH TO ENGINEERING DESIGN
}

\author{
Karia, Deval; Nambiar, Rohit S; Arora, Manish \\ Indian Institute of Science Bangalore
}

\begin{abstract}
Continuous subcutaneous insulin infusion using an insulin pump has been documented to have significant clinical benefits as a treatment plan for type 1 diabetes mellitus patients. However, despite this, adoption in resource constrained settings like India is severely limited. This is often attributed to the costs associated with the device.

The usage of such a device is closely associated with a patient's lifestyle and its design demands a more user-centric approach to engineering design. The stakeholder involvement is needed for validation and guiding the product development direction. In this paper, we've outlined a user-in-the-loop approach to engineering design using the design of an insulin pump as a case study.
\end{abstract}

Keywords: Biomedical design, User centred design, Design process, User-in-the-loop, Insulin pump

Contact:

Karia, Deval

Indian Institute of Science Bangalore

Centre for Product Design and Manufacturing

India

devalkaria@iisc.ac.in 


\section{INTRODUCTION}

Type 1 Diabetes Mellitus (T1DM) is a medical disorder that results from a failure of the patient's pancreas to produce insulin. It is thought to be an auto immune disease, wherein the body's immune system mistakenly attacks the beta cells (insulin producing cells) in the pancreas. This condition cannot be prevented. Insulin treatment is a critical component of a diabetes treatment plan for people with T1DM. Current-day insulin treatment plans are designed to imitate how the nondiabetic pancreas works. These plans are referred to as intensive insulin treatment and are recommended for most people with the disease (Hirsch et al., 1990). The patient, however, needs to administer three or more insulin injections (Multiple Daily Injections (MDI)) per day with frequent pricks to check blood sugar. Insulin pump therapy, also known as Continuous Subcutaneous Insulin Infusion (CSII), involves wearing a device (insulin pump) which provides a steady stream of insulin into the body. The insulin delivery from insulin pumps can more closely mimic what the body does naturally, thereby improving blood sugar control (Battelino et al., 2006). Consequently, a more flexible lifestyle can be afforded. The pumps, however, still require significant input from the patients.

Numerous additional studies have extensively documented the benefits of CSII over MDI as an insulin therapy for T1DM patients (Doyle et al., 2004, Retnakaran et al., 2004). Despite such overwhelming evidence in support, adoption of CSII is sparse, particularly in resource constrained settings like India. It is estimated that less than 5\% of T1DM patients use insulin pumps to manage their diabetes. Institutional factors aside, fixed and recurring costs of such a device are often found to be a withholding factor to widespread adoption (Kesavadev et al., 2010), with each device costing upwards of 2.5-3 lakh INR (3500 - 4200\$). However, this end goal of affordability should not be misinterpreted as merely engineering intervention. Not engaging relevant stakeholders in the engineering design process can lead to propagation of such misconstrued assumptions, leading to development of devices that may not be adopted by users. An in-depth understanding and assessment of location-specific needs can help ensure that more appropriate devices reach people in need (Sinha et al., 2011).

\subsection{Background and prior art}

Syringes and pens are presently the most popular insulin delivery devices in India. Though available for more than two decades, insulin pumps are now finding relevance because of their unique ability to continuously infuse insulin (Kesavadev et al., 2010). Unlike insulin shots with syringes, pump infusion sites need to be changed less frequently (once every 3 days). Documented evidence also points to the benefit of pumps in improving quality of life (Rubin et al., 2009). While there have been studies as early as the 1980s, demonstrating the benefits of using an insulin pump in Indian patients in a hospital setting (Mohan et al., 1983), the first pumps were introduced only around 1996. The current pumps in the Indian market are sophisticated devices, some of which are capable of functioning in a hybrid closed-loop. However, Medtronic Plc. (India Medtronic Pvt. Ltd.) is the only commercial player at the time of authoring this paper. This is in stark contrast to markets in in the United States, Europe and Australia, wherein patients have multiple options available: CellNovo, Insulet's Omnipod, Accu Check Spirit Combo, J\&J's Animas Vibe among others. Typically, these markets also have private insurance providers that cover all/a significant fraction of fixed and recurring costs associated with CSII. Peripherals such as a Continuous Glucose Monitor (CGM) are also often provided for by government programs (National Diabetes Services Scheme, Accessed October 2018).

Shape Memory Alloy (SMA) based actuators are used in the OmniPod insulin pump (not available in the Indian market) which is priced competitively at $30 \$$, however, it is designed to last for 3 days and the entire pump must be disposed of thereafter (Zisser, 2010). Most other commercially available pumps rely on a lead screw/nut coupled with a precision micro-motor to achieve minimal motion. Such motors, while being reliable are prohibitively expensive, they can cost upwards of 50-60k INR (700 - 800\$) for a single motor, with a planetary gear train to achieve internal speed reduction. These gear trains make use of micro-gears which are inherently expensive to manufacture, contributing significantly to the final cost of a pump. Each approach is prohibitive in terms of cost. However, as Chao et al. (2014) points out, this a setback to availability of products in resource constrained settings. In contrast, open-source hardware has been shown to allow medical devices to be built at 5-10\% of the cost of an equivalent commercially available device, with additional value added in terms of annual savings (Niezen et al., 2016). 
Development of an insulin pump from the ground up is a multi-faceted challenge, demanding significant engineering effort. However, this doesn't discount the nature of its usage, which is closely associated with a patient's lifestyle and culture. This demands a shift in design philosophy to allow for rigorous engagement of the user in the Engineering Design (ED) process.

\subsection{Research gaps and objective}

The traditional approach to ED, while being highly iterative only provides for stakeholder interaction in the initial research/task clarification stage. They are largely excluded in the subsequent development stages (Pahl et al., 2013). This, notwithstanding the body of evidence which shows that medical device adoption is subject to satisfaction of user needs and their ability to holistically fit into complex clinical settings (Yock et al., 2015). Industry regulations have reflected this need of user engagement in the design of medical devices (US Food and Drug Administration, 1997). However, there has not been a corresponding improvement in availability of practical guidelines demonstrating the need to iteratively include the user throughout the design process.

The objective of this paper is to address this need by presenting a case study, wherein a 'user-in-theloop' approach to ED has been adopted to design an insulin pump for resource constrained settings.

\section{RESEARCH METHODOLOGY AND APPROACH}

Design approaches that focus on the user have been discussed by various researchers using different terms such as human-centred design, customer-centric design, user-oriented design and user-centred design (UCD) (Veryzer et al., 2005). Most current versions of UCD have their origins in the seminal work of Norman and Draper (1986). UCD is defined as "an approach to designing ease of use into the total user experience with products and systems." (Vredenburg et al., 2002). This stands in contrast to a traditional approach, such as ED, particularly with respect to the logical order in which development is followed by user validation. In the UCD approach, development of only user validated designs is undertaken (Vredenburg et al., 2002), allowing for design changes at no extra cost. A change in the later stages of design can lead to a multi-fold increase in the corresponding cost (Fricke et al., 2000). In lieu of this, a 'user-in-the-loop' approach to ED has been adopted, with stakeholder(s) being engaged at the end of each stage to validate and modify, if necessary, the direction of product development (Figure 1). It is important to note that within the context of this case study, a user has been referred to as one of the many stakeholders present within the ecosystem.

The underlying ED process is an iterative one with four key stages: task clarification, conceptual design, embodiment design followed by detailed design/prototyping and testing. It is cyclic with regards to activities within each stage. The 'user-in-the-loop' approach imbibes user involvement while performing these activities. In the subsequent sections, the stages of this approach have been elucidated. A case study utilizing this approach to design an affordable insulin pump for T1DM patients is illustrated to further the discussion.

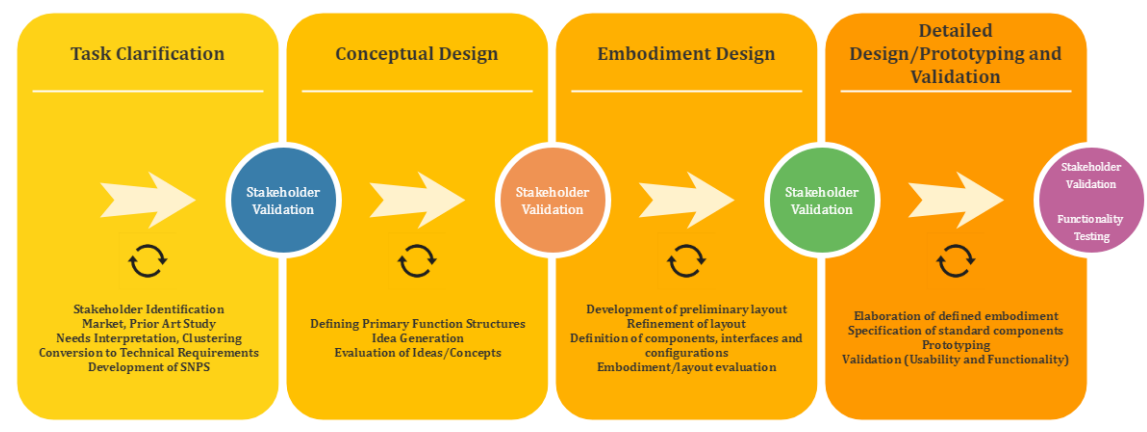

Figure 1. User-in-the-loop approach to engineering design

\section{STAGE 1: TASK CLARIFICATION}

The process begins with task clarification, involving identification of key stakeholders within the ecosystem, their needs and other key characteristics such as their financial capacity. It also involves a 
market and prior art study to identify key commercial players and their products. Subsequently, perceived needs are interpreted, clustered (stakeholder requirements) and converted into technical requirements by means of competitive benchmarking and the House of Quality (HoQ) methodology, sorted per their relative importance. A derived Solution Neutral Problem Statement (SNPS) serves as an input to the following stage.

A literature review was undertaken to gauge the relevance of insulin pumps as a delivery channel. Alternate mechanisms for delivery, such as vaporizers for inhaling the drug, despite being commercially available were severely limited in their scope and applicability. Other mechanisms in development were not found to not be technologically mature (Shah et al., 2016). The team concluded that CSII will remain the standard of care for the near future, until alternative technologies match the maturity, usability and convenience associated with pumps.

In order to gain a deeper understanding of the issues, the following stakeholders within the ecosystem were identified and interacted with: (1) T1DM patient, (2) patient parent(s)/immediate relatives, (3) individuals from the patients workplace/school, (4) doctor (endocrinologist), (5) nurses/pump trainer, (6) pump manufacturer/company representative, (7) pump salesman. The broad category of a T1DM patient is refined based on their insulin treatment plan (MDI vs. CSII), with MDI patients being further segregated based on their age (0-9, 9-19 and more than 19 years of age) and CSII patients segregated based on their lifestyle (sport enthusiast vs. sedentary). User personas were developed for each refined sub-category, to allow delving into specific inhibitions, concerns and issues faced by patients. Emphasis was laid on MDI patients and their reservations in migrating to CSII treatment. This was particularly important, since patients on MDI treatment are the primary target users for an affordable insulin pump. Among other concerns, it was understood that there is quite a lot of stigma associated with T1DM in India. Consequently patients, particularly women, tend to hide the pump under clothing to prevent discovery and make use of a washroom every time they need to administer a bolus dosage or monitor delivery.

A total of 14 stakeholder requirements (interpreted and clustered) were translated into 36 technical requirements. Table 1 illustrates top 6 stakeholder requirements (of the 14 identified) ranked in diminishing order of importance. Costs being affordable i.e. Affordability is found to be the critical non-clinical requirement. This is consistent with the findings of Jagtap et al. (2014). A corresponding Design Input Document (DID) was developed classifying these technical requirements into broad categories such as functional, hardware, security etc. which was then validated by key stakeholders.

\section{Table 1. Top 6 stakeholder requirements (interpreted, clustered and ranked)}

\begin{tabular}{ll} 
Rank & List of Stakeholder Requirements \\
\hline $\mathbf{1}$ & Extremely precise insulin delivery as dictated by medical requirements \\
$\mathbf{2}$ & Malfunctioning of the device in extreme environments must be informed to the user \\
$\mathbf{3}$ & Adequate alarms/response to Hypo-glycaemic and Hyper-glycaemic episodes \\
$\mathbf{4}$ & Device performance should be reasonably independent of external environmental conditions \\
$\mathbf{5}$ & $\begin{array}{l}\text { Provision to vary the Basal delivery rate across the day } \\
\mathbf{6}\end{array}$ \\
$\begin{array}{l}\text { The initial investment on the pump and recurring costs must be affordable to a majority of the } \\
\text { Total Available Market (TAM) }\end{array}$
\end{tabular}

\section{STAGE 2: CONCEPTUAL DESIGN}

Primary functions that the device must fulfil are defined and ideas to achieve each of those functions are conceptualized. Quick calculations, rapid prototyping, reverse engineering and literature reviews are typically used to evaluate brainstormed ideas. A set of feasible ideas to attain each function then serve as an input to development of the device embodiment. In the user-in-the-loop approach, key stakeholders are often consulted to answer queries pertaining to concept development/refinement or to gauge feasibility.

Based on a function structure of the device, three key functions were identified for brainstorming ideas: (1) mechanical actuation to precisely drive insulin into the tissue, (2) detecting hindrances in delivery (occlusion detection), (3) mounting the cannula into the subcutaneous tissue.

Brainstormed ideas for driving insulin into the tissue were broadly divided into two categories: micromechanism based, micro-pump based. Ideas in the latter category were largely discarded based on a literature review. These included technologies such as piezoelectric actuation, thermo-pneumatic/peristaltic 
micro-pumps. They were found to be not technologically mature or prohibitively expensive for the flow rates required (Nuxoll, 2013). Ideas in the former category were sketched and prototyped to gauge feasibility. Subsequently, a conceptualized mechanism was selected for further refinement. Technologies to detect occlusion in the fluid pathway were brainstormed, collated from literature, which were then tested.

\subsection{Reverse engineering: understanding flow rates}

Reverse engineering can be an effective technique when other solutions are available on the market. The exercise is not concerned with creating a copy or changing the artefact in some way; it is only an analysis to deduce design features from existing products (Chikofsky et al., 1990).

In order to understand the nature of insulin pump flow rates, reverse engineering of a commercially available pump was undertaken. Insulin delivery in CSII treatment is delivered in two modes: Basal and Bolus. A Basal rate is the uniform dosage maintained throughout the day, while a Bolus dose refers to delivery of a relatively higher dose of insulin over a brief period of time, typically administered by the patient before food intake. Commercially available pumps typically allow for Basal rates beginning from $0.05 \mathrm{IU} /$ hour (International Units per hour, $0.5 \mu \mathrm{L}$ per hour) to $25 \mathrm{IU} /$ hour $(250 \mu \mathrm{L}$ per hour), in increments of 0.05 IU/hour. With a typical insulin reservoir housing $3 \mathrm{~mL}$ (3000 $\mu \mathrm{L})$ of the drug, it can be estimated that incredible precision in actuation is required to deliver a volume of just $0.5 \mu \mathrm{L}$ every hour. This is compounded when a portion of this volume is delivered every minute or second of the stipulated hour. The clinical perspective pertaining to frequency of delivery was limited to its effects on plasma insulin levels (Chan et al., 2008). However, when an insulin pump is set to deliver insulin at a certain Basal rate (measured in IU/hour), does the delivery occur in a quantum dose every second or minute of the hour? This question was put forth to doctors and pump trainers. However, this largely being relevant to device engineering, stakeholders were unable to offer a perspective. Further investigations were carried out experimentally to understand the insulin delivery patterns.

The tip of an infusion set cannula was put under a microscope, with the pump set to deliver basal rates (Figure 2). Dyed water, in place of insulin was used to improve visibility. It was observed that the fluid was delivered in short acting pulses. When the total number of pulses were cross-referenced with the set Basal rate, for separate set Basal rates, it was determined that the volume quantum across different basal rates remains the same, with the time between pulses varying with Basal rates. As an example, a pump set to deliver at $1 \mathrm{IU} /$ hour will deliver 20 pulses of $0.05 \mathrm{IU}$ each with 3 minutes between each pulse, correspondingly for a $2 \mathrm{IU} /$ hour Basal rate, there will be 40 pulses of $0.05 \mathrm{IU}$ each, with 1.5 minutes between each pulse. A similar pattern of volume quantization is observed for a Bolus dose, however, the time between pulses was observed to be 2 seconds, owing to the brief time within which the total Bolus dose must be delivered.
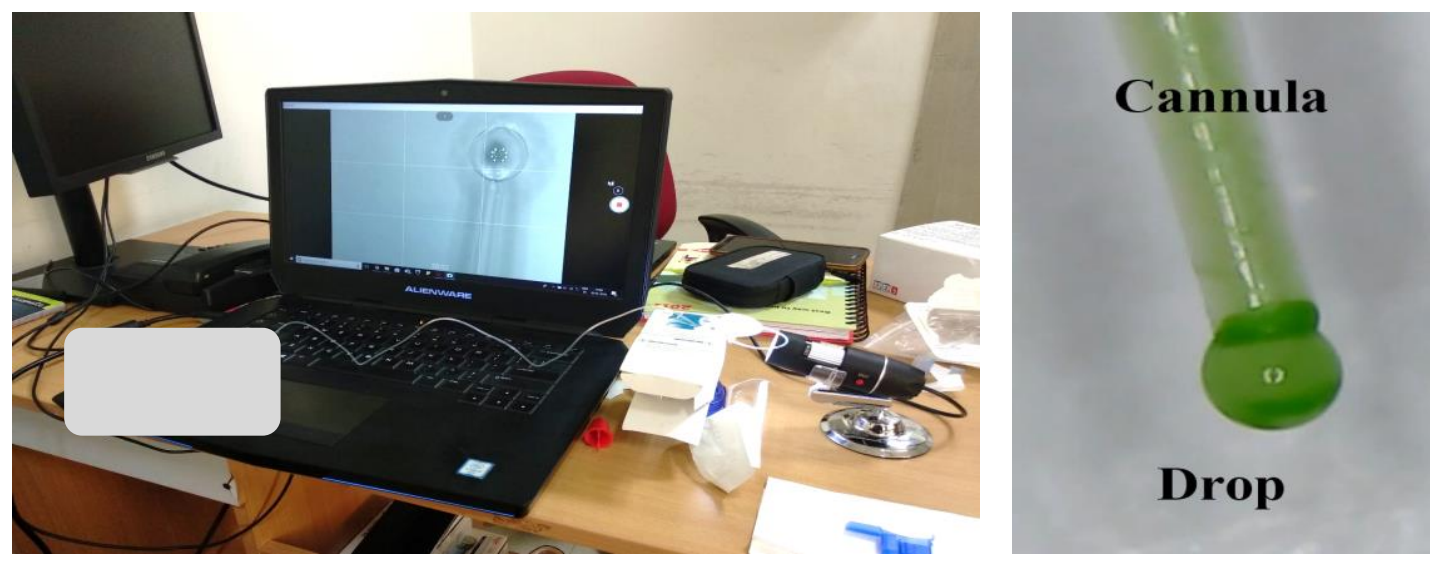

Figure 2. Experiments to understand delivery rates in commercially available pumps; Cannula tip as observed under a microscope 


\subsection{Experiments for occlusion detection}

Insulin pump occlusion can occur gradually because of progressive factors (insulin fibrils at the cannula outlet, progressive kinking of the cannula, compression of the skin around the infusion site) or can occur acutely. There are two main risks associated with a pump occlusion: (1) hyperglycaemia due to failure of insulin delivery; and (2) hypoglycaemia arising from partial occlusion, wherein if the infusion rate is increased to compensate for occlusion and maintain normoglycemia, then once the occlusion is relieved, a relatively large volume of non-infused insulin will be suddenly delivered (Klonoff et al., 2017). Each of these scenarios are significantly detrimental to patient health, and hence pumps must be capable of detecting occlusion and alerting the patient to take corrective action.

Multiple techniques can used for occlusion detection in a fluid pathway, these include force/pressure sensors, custom strain gauges among others (Olsen, 1997, DelCastilio et al., 2011). Motors/actuators within the fluid pathway experience a spike in current when the flow of fluid they are driving is occluded. This has been reported in literature (Davis et al., 1998), and stems from an increased load acting on the motor correlated to the degree of occlusion. Based on a broad survey of available sensors and their prices, motor current sensing was deemed to be a feasible option.

A simple open-source 3D printed syringe pump setup (Wijnen et al., 2014) with a Hall effect based current sensor was used to determine the load current through the motor. Occlusion was simulated by manually blocking the fluid pathway. It was observed that the current value spikes when this is done (Figure 3). Further, the sensor readings could be calibrated to detect the degree of occlusion as well.

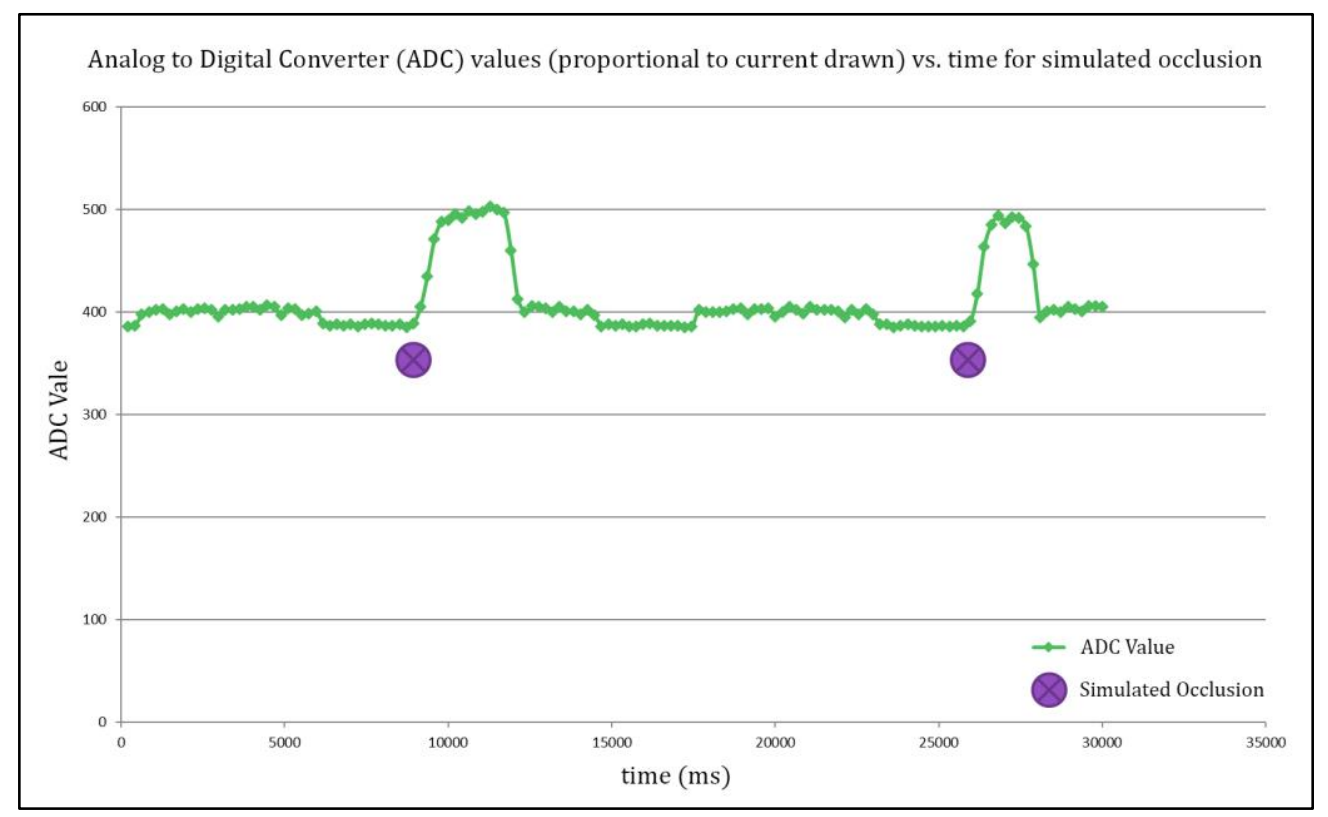

Figure 3. Spikes in current drawn for simulated occlusions in a fluid pathway

Based on exercises undertaken in the conceptual design stage, an approach was adopted to distribute the requisite speed reduction in multiple stages, with an acceptable compromise on the product size. The system being actuated by a nominal D.C. geared motor, a novel mechanism of converting continuous rotary input to intermittent output and a lead screw output to move the reservoir plunger. The developed kinematic chain was feedback controlled to ensure volumetric accuracy of the delivered drug.

\section{STAGE 3: EMBODIMENT DESIGN}

The embodiment design stage serves to bridge the gap between conceptual design and detailed design, particularly when the product is relatively complex, involving several sub-systems. It begins with the development of a preliminary layout to identify and position key system modules, following which the layout is further refined by definition of key physical components, interfaces and their geometric configurations. Aesthetic considerations are also considered at this point. Typically, there are multiple 
competing layouts for a particular concept, which are then evaluated to select one for further development. This assessment is largely done based on the designer's judgement. However, with the user-in-the-loop approach, competing layouts are taken to the user for evaluation and selection. This should ideally be done with physical/form mock-ups to aid the user.

Based on the conceptualized approach in the previous stage, two major embodiment layouts emerged: (1) infusion set based, (2) patch based. Each layout was detailed and then taken to stakeholders for evaluation.

\subsection{Embodiment selection: patch vs. infusion set}

An infusion set pump is broadly two part: the pump body and a disposable infusion set (Figure 4). The display, screen and user controls are all placed onto the pump body. The set tube is long and has a cannula at its end, which is mounted onto the body with the help of a standalone inserter. The pump body is typically kept in the patient's pocket, mounted onto a belt or under clothing via a separate accessory to conceal it. A remote to control the pump serves to help in concealment.

The patch pump embodiment has gained prominence with Insulet's OmiPod pump. The delivery device is a small patch, slightly larger than a wrist watch dial. It does not house a display unit or controls, which is done with a separate device via wireless communication. The infusion cannula is self-inserting by means of a pre-tensioned spring. The delivery device in the OmniPod pump is designed to function for three days and must be disposed of thereafter. The embodiment layout developed by our team, however, allowed for reuse, albeit with a minor compromise on size (Figure 4).

Both embodiments, their detailed models and form mock-ups for the pump were conveyed to the stakeholders. The broad consensus among them favoured an infusion set embodiment, particularly because a pump detached from the infusion site would allow them to store it discreetly as per their convenience. A small portion of the stakeholders were also apprehensive about having a larger patch attached to their body.
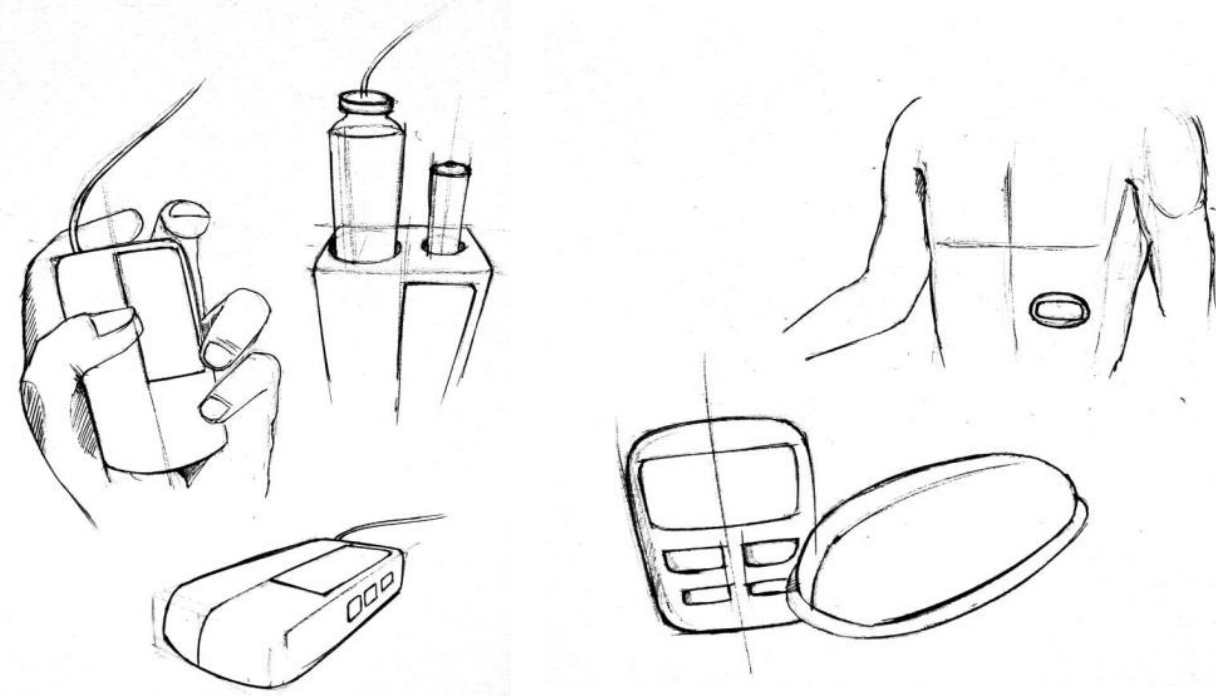

Figure 4. Developed embodiments: Infusion set vs. Patch based

In discussions pertaining to embodiment selection, the issue of infusion tube entanglement/accidental detachment cropped up multiple times. It was then proposed to be tackled with an anchor point to hold excess tubing, which can be unwound as required.

The embodiment design stage concluded with selection of the infusion set based embodiment and its refinement by definition of aspects such as the anchor point, kind of gearbox, insulin reservoir viewer among others.

\section{STAGE 4: DETAILED DESIGN/PROTOTYPING AND TESTING}

Each aspect of the defined embodiment is further elaborated, prominently by 3D CAD models. Specifications for standard components are defined and materials required for prototyping are 
procured. Simulations are also undertaken, if required, to define dimensions of critical components. While quick prototyping is undertaken in the previous stages as well, prototyping at this stage is undertaken for the purpose of product realization and not to evaluate options for a function. This involves significant consideration of fits and tolerances. Testing broadly pertains to checking whether the solution meets the goals of usability and functionality. The former is evaluated based on stakeholder feedback, while the latter is evaluated basis the fulfilment of technical requirements defined in the task clarifications stage.

\subsection{Prototyping}

CAD models of components derived from detailed design of the embodiment served as the backbone of prototyping activities using multiple processes. The gear train, encoder coupling and the base structure to mount all components were 3D printed with a Stratasys Objet 500V Polyjet printer. Requisite electronic components such as the current sensor, microcontroller etc. were purchased as breakout boards derived from open-source designs. This decision was important to allow for eventual release of the insulin pump as an open-source medical device. The outermost enclosure was CNC milled to achieve a brushed metal texture, with the top screen made from translucent acrylic to provide an edge to edge finish (Figure 5).

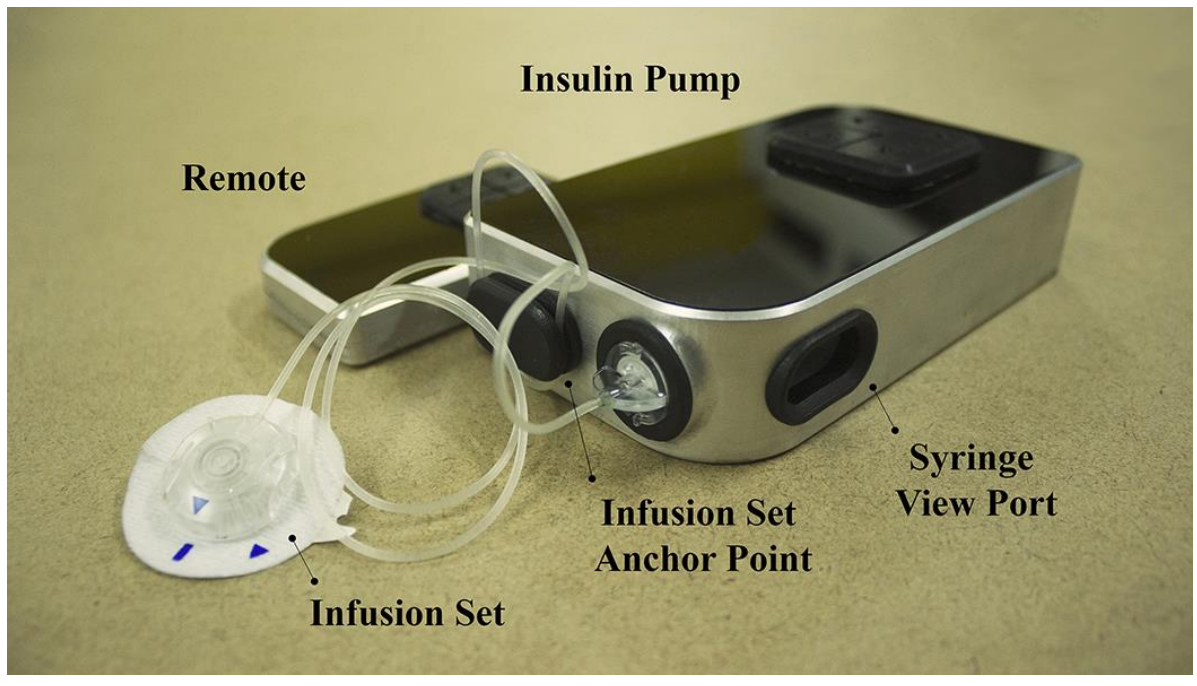

Figure 5. Prototype insulin pump and remote

\subsection{Testing and validation}

The testing and validation of the developed pump was two pronged. The first involved measurement of volume delivered for a given input dose. The second involved usability validation undertaken with the stakeholders.

In order to test the amount of fluid delivered by the device at each pulse, a pre-set dosage was delivered onto a micro-weighing scale with a resolution of $0.1 \mathrm{mg}$ (equivalent to $0.1 \mu \mathrm{L}$ or $0.01 \mathrm{IU}$ ). The readings were recorded, and the value delivered per pulse was determined. The same exercise was repeated for a commercially available pump, and the delivery volumes compared (Figure 6). It was observed that the prototype pump was not as accurate as a commercially available one. Additionally, it had an erratic delivery bias in contrast to a consistent negative bias provided by the commercial one, which is also clinically preferred to avoid unwarranted risks of a hypoglycaemic episode.

Stakeholders appreciated the aesthetic appeal, ergonomics and the broad motivation behind the development of an affordable pump for resource constrained settings such as India. Patients and clinical experts highlighted the need for further refinement of the user interface flow to make it risk averse and comprehensive, the bias by design to right-handed individuals and the larger size compared to a commercial pump. The clinical need for higher accuracy and a negative delivery bias was also strongly emphasized. 


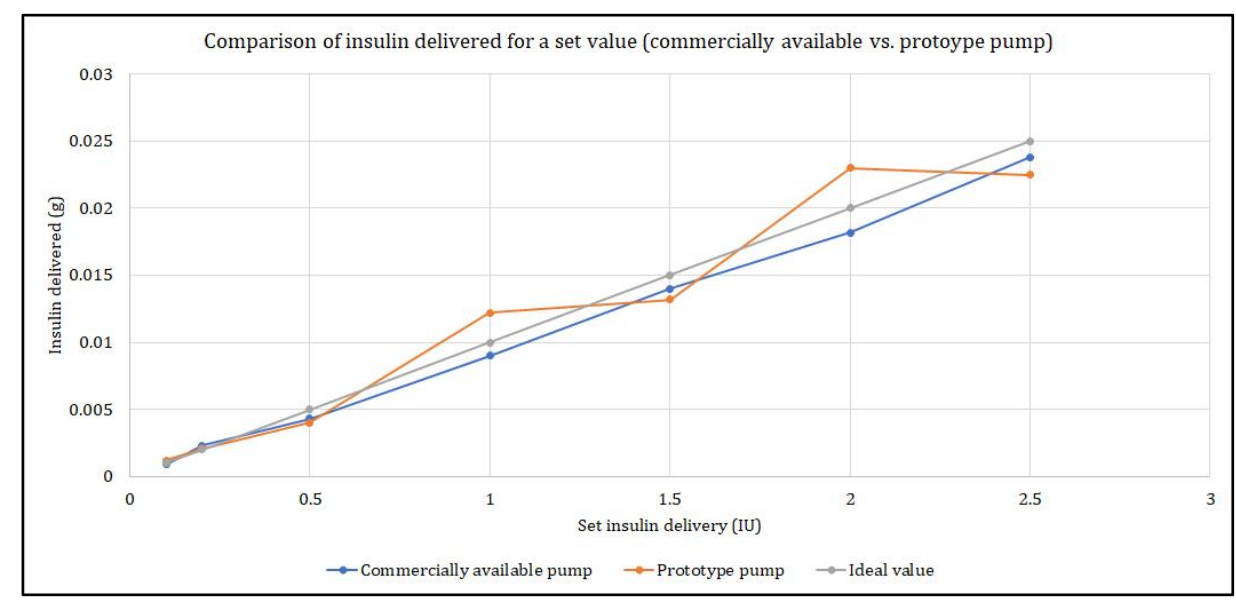

Figure 6. Comparison of fluid volume delivered for a set input dose (commercially available pump vs. prototype pump

\section{DISCUSSIONS AND CONCLUSION}

This paper has outlined the 'user-in-the-loop' approach in the design of biomedical devices and presents a case study on the design of an insulin pump for resource constrained settings. The approach allows for validation/reorientation of the product development direction at critical junctures. An example of this is the embodiment selection i.e. user input led to the selection of an infusion set based embodiment. In a traditional approach, this decision would have been taken by a designer, which may not have been necessarily conducive to the user.

The use of distributed speed reduction coupled with the use of the conceptualized novel mechanism is aimed at avoiding the use of an expensive precision D.C. motor/encoder. This is expected to bring down the fixed cost significantly, which was the most critical non-clinical requirement.

The open-source communities around the Artificial Pancreas (AP) system are active and well established (Open Artificial Pancreas System, Accessed January 2018). However, they are limited to open-source software algorithms relying on firmware flaws in a commercially available pump for insulin delivery. The developed pump could contribute to the hardware aspect of this community and complete a vital link in the triad (Insulin Pump, AP Algorithm, CGM). Such an unconventional approach to clinical adoption would allow multiple innovators/institutions the freedom to operate and test the device independently, potentially increasing safety, security and robustness. Such outcomes for open-source projects have been documented in literature as well (Niezen et al., 2016). It is, however, important to note that for the eventual release of a device into the open-source domain, designers must plan and preferentially base selection of building blocks/components on existing opensource designs. These decisions should be taken during the earlier stages of design.

\section{REFERENCES}

Battelino, T. (2006), "Risk and benefits of continuous subcutaneous insulin infusion (CSII) treatment in school children and adolescents", Pediatric Diabetes, Vol. 7, pp. 20-24.

Chan, A., Breton, M.D. and Kovatchev, B.P. (2008), "Effects of pulsatile subcutaneous injections of insulin lispro on plasma insulin concentration levels”, Journal of Diabetes Science and Technology, Vol. 2 No. 5 , pp. 844-852.

Chao, T.E., Lo, N.C., Mody, G.N. and Sinha, S.R. (2014), "Strategies for last mile implementation of global health technologies", The Lancet Global Health, Vol. 2 No. 9, pp. e497-e498.

Chikofsky, E.J. and Cross, J.H. (1990), "Reverse engineering and design recovery: A taxonomy”, IEEE Software, Vol. 7 No. 1, pp. 13-17.

Davis, D.L., Hatch, R.F., Poirier, D.A. and Patient Solutions Inc (1998), "Method of measuring an occlusion in an infusion device with disposable elements". U.S. Patent 5,803,712.

DelCastilio, J.A., Yardimci, A., Baxter Healthcare SA and Baxter International Inc (2011), "System and method for detecting occlusion using flow sensor output”. U.S. Patent 7,880,624.

Doyle, E.A., Weinzimer, S.A., Steffen, A.T., Ahern, J.A.H., Vincent, M. and Tamborlane, W.V. (2004), “A randomized, prospective trial comparing the efficacy of continuous subcutaneous insulin infusion with multiple daily injections using insulin glargine”, Diabetes Care, Vol. 27 No. 7, pp. 1554-1558. 
Fricke, E., Gebhard, B., Negele, H. and Igenbergs, E. (2000), "Coping with changes: causes, findings, and strategies", Systems Engineering, Vol. 3 No. 4, pp. 169-179.

Hirsch, I.B., Farkas-Hirsch, R. and Skyler, J.S. (1990), "Intensive insulin therapy for treatment of type I diabetes", Diabetes Care, Vol. 13 No. 12, pp. 1265-1283.

Jagtap, S., Larsson, A., Hiort, V., Olander, E., Warell, A. and Khadilkar, P. (2014), "How design process for the base of the pyramid differs from that for the top of the pyramid", Design Studies, Vol. 35 No. 5, pp. 527-558.

Kesavadev, J., Das, A.K., Unnikrishnan, R., Joshi, S.R., Ramachandran, A., Shamsudeen, J., Krishnan, G., Jothydev, S. and Mohan, V. (2010), "Use of insulin pumps in India: suggested guidelines based on experience and cultural differences", Diabetes Technology \& Therapeutics, Vol. 12 No. 10, pp. 823-831.

Klonoff, D.C., Freckmann, G. and Heinemann, L. (2017), Insulin pump occlusions: for patients who have been around the (infusion) block.

Mohan, V., Shyamsunder, R., Ramchandran, A., Snehalatha, C. and Viswanathan, M. (1983), "Experience with insulin pump treatment in Indian diabetics. A preliminary report", The Journal of the Association of Physicians of India, Vol. 31 No. 11, pp. 715-717.

National Diabetes Services Scheme. (2018), “Continuous Glucose Monitoring”. [ONLINE] Available at: https://www.ndss.com.au/cgm. [Accessed October 2018]

Niezen, G., Eslambolchilar, P. and Thimbleby, H. (2016), “Open-source hardware for medical devices”, BMJ Innovations, Vol. 2 No. 2, pp. 78-83.

Norman, D.A. and Draper, S.W. (1986), User centered system design: New perspectives on human-computer interaction. CRC Press.

Nuxoll, E. (2013), "BioMEMS in drug delivery”, Advanced Drug Delivery Reviews, Vol. 65 No. 11-12, pp. 1611-1625.

Olsen, J.M. and Deltec Inc (1997), “Occlusion detection system for an infusion pump”. U.S. Patent 5,695,473.

Open Artificial Pancreas System. (2018), "What is \#OpenAPS?”. [ONLINE] Available at: https://openaps.org. [Accessed January 2018]

Pahl, G. and Beitz, W. (2013), Engineering design: a systematic approach. Springer Science \& Business Media.

Retnakaran, R., Hochman, J., DeVries, J.H., Hanaire-Broutin, H., Heine, R.J., Melki, V. and Zinman, B. (2004), "Continuous subcutaneous insulin infusion versus multiple daily injections: the impact of baseline A1c", Diabetes Care, Vol. 27 No. 11, pp. 2590-2596.

Rubin, R.R., Peyrot, M., Kruger, D.F. and Travis, L.B. (2009), "Barriers to insulin injection therapy", The Diabetes Educator, Vol. 35 No. 6, pp. 1014-1022.

Shah, R.B., Patel, M., Maahs, D.M. and Shah, V.N. (2016), "Insulin delivery methods: Past, present and future", International journal of pharmaceutical investigation, Vol. 6 No. 1, p. 1.

Sinha, S.R. and Barry, M. (2011), "Health technologies and innovation in the global health arena", New England Journal of Medicine, Vol. 365 No. 9, pp. 779-782.

US Food and Drug Administration, (1997), Design control guidance for medical device manufacturers. Center for Devices and Radiological Health.

Veryzer, R.W. and Borja de Mozota, B. (2005), "The impact of user-oriented design on new product development: An examination of fundamental relationships", Journal of Product Innovation Management, Vol. 22 No. 2, pp. $128-143$.

Vredenburg, K., Isensee, S., Righi, C. and University of Texas at Austin/Software Quality Institute, (2002), User-Centered Design: An Integrated Approach. Prentice Hall PTR, Upper Saddle River NJ.

Wijnen, B., Hunt, E.J., Anzalone, G.C. and Pearce, J.M. (2014), “Open-source syringe pump library”, PloS One, Vol. 9 No. 9, p. e107216.

Yock, P.G., Zenios, S., Makower, J., Brinton, T.J., Kumar, U.N., Watkins, F.J., Denend, L., Krummel, T.M. and Kurihara, C.Q. (2015), Biodesign: the process of innovating medical technologies. Cambridge University Press.

Zisser, H.C. (2010), “The OmniPod Insulin Management System: the latest innovation in insulin pump therapy”, Diabetes Therapy, Vol. 1 No. 1, pp. 10-24.

\section{ACKNOWLEDGMENTS}

We acknowledge the funding from the Indian Council of Medical Research (ICMR) as part of the IMPRINT Program of the Ministry of Human Resource Development, Government of India. Professor Radhakant Padhi, Department of Aerospace Engineering, Professor KVS Hari, Department of ECE, Indian Institute of Science (IISc) for their feedback during the design process and Dr. Shakuntala Acharya for her critical reviews of the design approach undertaken.

The following clinics/centres/departments were involved in the task clarification/testing: Department of Endocrinology, Ramaiah Memorial Hospital (Karnataka, India); Jnana Sanjeevani Diabetes Centre (Karnataka, India); Optics and Microfluidics Instrumentation (OMI) Lab, Department of Instrumentation and Applied Physics, IISc. 